\title{
Verification, Validation, and Accreditation (VV\&A) Considering Military and Defense Characteristics
}

\author{
Jung Hoon Kim, Seugmin Jeong, Sunkyung Oh, Young Jae Jang* \\ Industrial and Systems Engineering, KAIST, Daejoen, Korea
}

(Received: February 18, 2015 / Revised: March 13, 2015 / Accepted: March 16, 2015)

\begin{abstract}
In this paper, we identify the characteristics of modeling and simulation (M\&S) for military and defense and propose the method of verification, validation, and accreditation (VV\&A) using the identified characteristics. M\&S has been widely used for many different applications in military and defense, including training, analysis, and acquisition. Various methods and processes of VV\&A have been proposed by researchers and M\&S practitioners to guarantee the correctness of M\&S. The notion of applying formal credibility assessment in VV\&A originated in software engineering reliability testing and the systems engineering development process. However, the VV\&A techniques and processes proposed for M\&S by the research community have not addressed the characteristics and issues specific to military and defense. We first identify the characteristics and issues of military/defense M\&S and then propose techniques and methods for VV\&A that are specific for military/defense M\&S. Possible approaches for the development of VV\&A are also proposed.
\end{abstract}

Keywords: Simulation, Modeling Validation and Verification, Military and Defense Simulation

* Corresponding Author, E-mail: yjang@kaist.edu

\section{INTRODUCTION}

With advances in computing and information technology, modeling and simulation (M\&S) has been widely applied in various areas in the military, and its role in the military and defense industry is particularly critical. For example, methods of measuring the performance of a newly developed weapon system are very limited unless the weapon system is actually tested against an enemy. Therefore, in practice, a model should be created to imitate the behavior of the weapon system and the environment in which it is to be used to test its performance. A simulation is then constructed based on the model. M\&S are used to generate data for the weapon system and to analyze its effectiveness. M\&S is also used to train personnel in the use of the weapon system. Due to the limitations of actual usability and performance evaluation in real situations, the military industry makes more extensive use of M\&S than any other industry, and military decisions and activities often depend on its use. In particular, $M \& S$ is considered to be crucial in training, analysis, and defense acquisition. Simulationbased training has been widely used from an individual training level to the campaign level, such as war-game training. Furthermore, M\&S has become an essential tool for tactical or strategic-level analysis. Mission-critical decisions are analyzed with simulation models before decisions are made. Furthermore, simulation has been common in the acquisition and deployment of weapons.

To guarantee that M\&S adequately imitates the key 
behaviors of the real system, validation and verification processes are used. Verification is defined as the process of ensuring that the implementation of the M\&S is correct. Validation is defined as the process of developing M\&S within a satisfactory range of accuracy. Baci (1994, 2002) defined validation and verification as follows "The validation is substantiating that the model, within its domain of applicability, behaves with satisfactory accuracy consistent with the study objectives. The validation deals with building the right model. The verification is substantiating that the model is transformed from one form into another, as intended, with sufficient accuracy." $\mathrm{He}$ emphasized that validation and verification is conducted by running the model under the same input conditions that drive the system and by comparing the behavior of the model with that of the system. This definition of validation and verification is widely accepted within the M\&S community (Jang et al., 2002; Tolk, 2012; Oberkampf et al., 2004).

The validation and verification process was originally used in software engineering to guarantee quality (IEEE Standard for Software Verification and Validation, 1998; Adrion et al., 1998). In addition to the validation and verification process, an additional process called accreditation certifies the fidelity of the M\&S. The validation, verification, and accreditation (VV\&A) process is performed to ensure that an M\&S meets its requirements and to guarantee its quality.

In this paper, we first summarize the previous research on VV\&A in software engineering and systems engineering (SE). We then introduce the characteristics of the military/defense $M \& S$ and propose a VV\&A method using the identified characteristics.

\section{VV\&A IN SOFTWARE ENGINEERING AND SE}

\subsection{VV\&A as a Process}

We first surveyed the literature to investigate the VV\&A processes proposed by the research community and those used in practice in the military/defense field. The National Aeronautics and Space Administration (NASA) in the United States was the first mover in the standardization of VV\&A by formalizing its use as a guideline in the M\&S process. The NASA standard document of M\&S specifies the overall steps and process of VV\&A; specifications can be found in (NASA-STD$7009,2008)$. These studies focus on organizational issues, domain knowledge issues, and decision steps in processing VV\&A. They specify the importance of military domain knowledge rather than processing methods in VV\&A to ensure the quality of the $M \& S$ based on the overall development process. That is, unlike a final qua- lity control of the development process, they emphasize that VV\&A should be embedded into the overall process from the early beginnings to the complete test stage.

\subsection{Software Engineering}

The origin of VV\&A is the quality assurance process in software engineering, in which verification is performed at each step of development to ensure that each step meets requirements. Validation is conducted throughout the development cycle to ensure that the final software performs as planned and meets the initial requirements. A separate testing process is also performed by evaluating the software with various scenarios and data (National Computerization Agency, 1990). Together, these processes in the development of software are called VV\&T (validation, verification, and testing; note that the term testing is used instead of accreditation). VV\&T is not just a process that is performed at the end of the development stage; it is rather a continuous process that is aligned with the entire lifecycle of software development. In software engineering, VV\&T is therefore considered developmental lifecycle management. The key concept is to achieve the efficient management of software development by alignment with the VV\&T process. The concept, approaches, and procedures of VV\&T aligned with software development can be found in (Godefroid, 2005; Godefroid and Khurshid, 2002; Wakeland et al., 2005). From these studies, we conclude that the notion of performing VV\&A throughout the entire development of $\mathrm{M} \& \mathrm{~S}$ definitely originated from the concept of VV\&T in software engineering.

\subsection{SE}

The M\&S process requires more than the generation of software code. The simulation should imitate the actual system and meet the intended requirements specified at the system design stage. That is, although a developed simulation may be an error-free program, it is useless if it does not correctly generate the physical interactions or activities in the simulated world. In addition, the level of abstraction and granularity of the simulation model must be aligned with its intended use. The real-world domain knowledge that the simulation is intended to imitate should be integrated with the simulation engineering process. Therefore, the overall M\&S process requires not only simulation or software engineering skill but also domain knowledge and other related expert skills. To incorporate a variety of skills and knowledge, the development process of M\&S has adopted the system design process proposed in SE.

According to the NASA Systems Engineering Handbook and INCOSE Systems Engineering Handbook (NASA Systems Engineering Handbook and INCOSE Systems 
Engineering Handbook), the verification and validation are defined such that "verification is to make sure that the systems is built to the target specification, and validation is to make sure the end product will perform as customers intended to perform in the environment." The definition itself does not have a sharp distingtion from the deficiton from other areas in cluding the quality assurance process in SE (IEEE Standard for Software Verification and Validation, 1998; Adrion et al., 1982; Choi, 2012).

However, one distintion of the definition in SE can be found in the "V" model. Specifically, the adoption of SE methods in the design process for VV\&A and M\&S can be explained well with the "V" model framework in SE, as shown in Figure 1. The framework consists of multiple processes depicted in quadrangles. The processes can be placed into two major categories: the definition and decomposition step and the integration and testing step. In the first step, the concept and requirements are defined at the system and subsystem levels and the detailed design is developed. After the first step is completed, the implementation process proceeds, followed by the validation and verification processes, i.e., the integration and test step.

This "V" framework in SE matches the concept of VV\&A well. In M\&S, the requirements of the simulations are defined, and the concept and design of the system follow. The definition and decomposition steps are critical in VV\&A because the validation and verification stages after implementation are performed on the basis of the concept definitions and requirements specified in the integration and test step. The concept of the framework aligns well with that of VV\&A and is therefore commonly used in VV\&A practice (Choi, 2012).

\section{MILITARY/DEFENSE M\&S CHARAC- TERISTICS}

In the previous section, we discussed the origins of VV\&A and the concept of VV\&A in the software engineering and SE fields. The concept of VV\&A in the military/defense industry was first specified in the 1990s, and the approaches and methods of VV\&A used in the military/defense industry are still being developed by many practitioners and researchers. It is generally agreed that the VV\&S process is crucial in M\&S in the military/defense industry, but no specific instructions or even best practices have yet been recognized. In this section, we identify the characteristics of M\&S in the military/defense industry before we propose directions of research to develop specific methods and approaches for military/defense VV\&A.

Military/defense M\&S is a hierarchical system, which distinguishes it from M\&S in other areas. Military/defense M\&S is divided into three hierarchical levels depending on the level of abstraction (Figure 1).

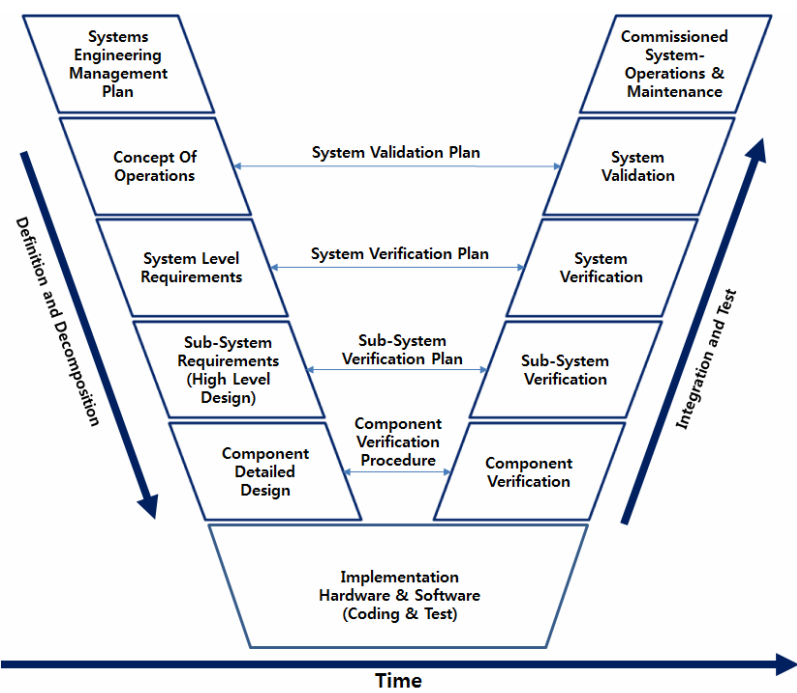

Figure 1. "V" model framework (BMDO Directive 5022, 1994).

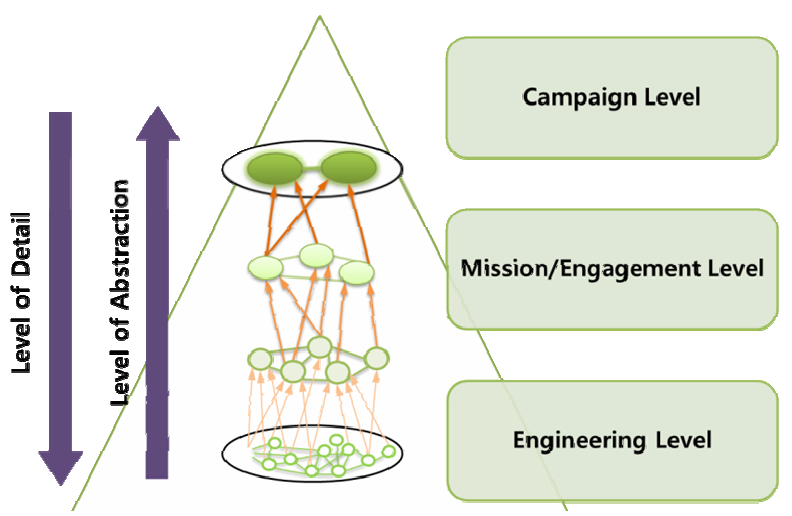

Figure 2. Level of abstraction in M\&S and the characteristics of each level.

\subsection{Engineering Level}

The lowest level of M\&S is the engineering level. M\&S at this level mostly simulates the physical movements of a weapon or an individual entity over time. For example, simulation of the trajectory of a missile, driven by a nonlinear differential equation, is categorized at the engineering level. Because the system is nonlinear, the missile's trajectory is estimated by simulation modeling. Commercial software such as Matlab or Simulink is widely used to run the numerical simulation models used at this level. The domain knowledge required for VV\&A at this level includes weapon engineering, mechanical engineering, SE, and numerical simulations. In the process of VV\&A, detailed analysis of the mathematical model and investigation of the numerical algorithm must be conducted.

\subsection{Mission/Engagement Level Engineering}

The second level of the hierarchy is the mission/ 
engagement level. M\&S at this level includes multiple weapons, soldiers, and systems. The M\&S must also simulate the environment, such as the weather, in which these entities operate. Because multiple entities must interact, the $M \& S$ engineers must determine a means for these entities to communicate. The M\&S utilizes an eventbased simulation or a combined event and numerical simulation. For example, an individual weapon in the simulation such as a missile may be modeled using a numerical simulation as at the engineering level. However, the interaction between the missile and the soldiers may be modeled using event-based simulation modeling. At this level, VV\&A needs to focus not only on the approaches used at the engineering level, but also on additional activities such as the validation of environmental data (e.g., weather data) and communication between the entities. Because the model becomes more complicated at the mission/engagement level, a certain level of abstraction is needed, and a more qualitative analysis of the VV\&A process is also expected.

A good example of the mission/engagement level in $\mathrm{M} \& \mathrm{~S}$ is the simulator developed for the medium-range surface-to-air missile system, ChunGung, developed by Korea's Agency for Defense Development (ADD) (Choi, 2012; Medium Range Surface to Air Missile System ChunGung, 2012). The ChunGung includes multifunction radar, an engagement control station, a missile launcher, and the missile itself. The simulator for the system must therefore include each of these entities. The missile dynamics and the interaction between these entities must be considered.

\subsection{Campaign Level}

The highest level in the hierarchy is the campaign level. A war-game simulation is a good example of a campaign-level M\&S. It aggregates the entities from the engineering and mission/engagement levels. In addition, the enemy behavior model and various scenarios regard- ing an enemy's reaction must be analyzed. Because the simulation model at this level deals with complicated interactions between entities, the VV\&A activities must also be well established. In addition, detailed technical analysis or objective evaluation of VV\&A may not be possible because of the greater level of abstraction. As a consequence, more subjective analysis should be performed in VV\&A. One widely used method is the Delphi method, a structured communication and opinion survey technique that was originally developed as a systematic, interactive method of forecasting or evaluation that relies on expert domain knowledge (Jang et al., 2002). A good example of the use of the Delphi method in VV\&A is the SpaceNet Simulation developed by NASA to evaluate supply logistics by space ships between the Earth and the moon (Ahn, 2007). Because the process of supplying material from the earth to the moon has not actually occurred, the data are limited. Objective and quantitative approaches of VV\&A are thus very difficult to apply. Therefore, subject experts are involved in the development process and continuously evaluate the development process for VV\&A.

\section{VV\&A DIRECTIONS}

As described in the previous section, military/defense $M \& S$ has different characteristics depending on the model's level of abstraction. The methods and approaches of VV\&A must therefore be designed on the basis of the model to which it belongs.

Table 1 describes the objectives, required skills, and methods of VV\&A for each level. Because M\&S at the engineering level deals with detailed entity-level simulation with mostly numerical methods, statistical and numerical analysis can be applied for VV\&A. The objective of VV\&A is the validation of the mathematical model and the verification of the codes of the simulation. A quantitative approach with statistical tests, analysis of

Table 1.

\begin{tabular}{|c|c|c|c|}
\hline Table 1. & Engineering Level & Mission/Engagement Level & Campaign Level \\
\hline Objective of VV\&A & $\begin{array}{l}\text { - Dynamic model validation } \\
\text { - Numerical simulation veri- } \\
\text { fication } \\
\text { - System analysis }\end{array}$ & $\begin{array}{l}\text { - Dynamic model validation } \\
\text { - Verification between entities } \\
\text { - Environment model validation } \\
\text { - Data validation }\end{array}$ & $\begin{array}{l}\text { - Strategy validation } \\
\text { - Verification on system integra- } \\
\text { tion }\end{array}$ \\
\hline Required Skills & $\begin{array}{l}\text { - Statistical analysis } \\
\text { - Numerical analysis } \\
\text { - Dynamic system modeling }\end{array}$ & $\begin{array}{l}\text { - Statistical analysis } \\
\text { - Event-based simulation verification } \\
\text { - Mission and engagement system } \\
\text { domain knowledge } \\
\text { - Military science } \\
\text { - Military tactic analysis }\end{array}$ & $\begin{array}{l}\text { - Military strategy } \\
\text { - Military defense scenarios } \\
\text { analysis } \\
\text { - System analysis }\end{array}$ \\
\hline $\begin{array}{c}\text { Proposed VV\&A } \\
\text { Methods }\end{array}$ & $\begin{array}{l}\text { - Statistical test } \\
\text { - ANOVA } \\
\text { - DOE }\end{array}$ & $\begin{array}{l}\text { - Statistical test } \\
\text { - ANOVA (Analysis of Variance) } \\
\text { - DOE } \\
\text { - System engineering analysis }\end{array}$ & $\begin{array}{l}\text { - Delphi } \\
\text { - AHP } \\
\text { - System engineering analysis }\end{array}$ \\
\hline
\end{tabular}


variance, and experimental design is recommended for VV\&A. For the mission/engagement level, the objective is extended to the validation of the data and the integration of the methods between entities. Quantitative and qualitative skills are required, and knowledge of military tactics and mission engagements is needed. The " $\mathrm{V}$ " model of SE analysis can be applied at this level. More high-level objectives and methods are needed at the campaign level because of the scale and degree of the abstraction. The primary goals of VV\&A include not only technical and quantitative validation and verification, but also strategic analysis. The required skills include knowledge of military strategy and a high-level understanding of military operations. As described in the previous section, the Delphi method is recommended for VV\&A. An analytic hierarchy process also can be used for the quantitative analysis of group decisions. Of couser, more rigoruse research on Dephi method for VV\&A still needs to be investigated. Consensus measurement plays an important role in Delphi process. The measurement has to be considered an important component of Delphi analyses and data interpretation However, numerous research reveals that there are obvious deficits in the practice and rigour of consensus measurement for Delphi research: mistakes in statistical tests or their premises have even been made (Heiko, 2012).

Another direction we propose is adding the risk analysis in the VV\&A process or using the VV\&A as a risk mitigation strategy. In particular, As M\&S are widely used, it becomes increasingly crutial to accumulate evidence that these tools are working correctly. VV\&A of models and simulations are required to mitigate the risk of incorrect decisions based on M\&S outputs. The goal, then, of VV\&A is to reduce the risk of poor decisions based on incorrect models and simulations (Kilikauskas and Hall, 2005).

\section{CONCLUSIONS}

In this paper, we discuss the concept of VV\&A and its origins in software engineering and SE. We then identify the characteristics of VV\&A by the abstraction level of $M \& S$ in the military/defence context. We categorize military/defense M\&S into three levels: engineering, mission/engagement, and campaign. We show that each level of M\&S requires different approaches and goals. VV\&A methods and approaches must therefore be developed based on the level of abstraction. VV\&A is crucial in military/defense, and the need for VV\&A has been well recognized. VV\&A is still in a developmental stage. We emphasize that the methods and approaches of VV\&A should progress according to the level of abstraction. More research on this approach is needed, and we suggest a case study of an actual VV\&A process to analyze the process according to the proposed categorized perspectives introduced in this paper.

\section{ACKNOLEDGEMENTS}

This work was supported by Defense Acquisition Program Administration and Agency for Defense Development under the contract UD140022PD, Korea.

\section{REFERENCES}

Adrion, W. R., Branstad, M. A., and Cherniavsky, J. C. (1982), Validation, Verification, and Testing of Computer Software, ACM Computing Surveys (CSUR), 14(2), 159-192.

Ahn, J. (2007), Pilot Study: Credibility Assessment of Spacenet 1.3 with NASA-STD-(I)-7009.

Balci, O. (1994), Validation, Verification, and Testing Techniques Throughout the Life Cycle of a Simulation Study, Annals of Operations Research, 53(1), 121-173.

Balci, O. Adams, R. J., Myers, D. S., and Nance, R. E. (2002), A Collaborative Evaluation Environment for Credibility Assessment of Modeling and Simulation Applications, Paper presented at the Simulation Conference.

Choi, Y. J. (2012), The Study of Process for VV\&A on Acquiring the Credibility of M\&S, Journal of the Korea Society of Systems Engineering, 5(2).

Godefroid, P. (2005), Software Model Checking: The Verisoft Approach, Formal Methods, System Design, 26(2), 77-101.

Godefroid, P. and Khurshid, S. (2002), Exploring Very Large State Spaces Using Genetic Algorithms, Tools and Algorithms for the Construction and Analysis of Systems, 266-280.

Heiko, A. (2012), Consensus measurement in Delphi studies: review and implications for future quality assurance, Technological Forecasting and Social Change, 79(8), 1525-1536.

IEEE Standard for Software Verification and Validation (1998), 1012-1998.

Jang, Y. J., Kim, J. H., Jo, H. J., Hwang, I. H., and Kim, D. Y. (2012), Research Direction Proposal on VV\&A of the Defense M\&S and the Categorization of VV\&A Method Based on Modeling Abstraction, Journal of the Korea Society for Simulation, 2.

Kilikauskas, M., and David, H. H. (2005), The use of $\mathrm{M} \& \mathrm{~S}$ VV\&A as a risk mitigation strategy in defense acquisition, The Journal of Defense Modeling and Simulation: Applications, Methodology, Technology, 2(4), 209-216.

Medium Range Surface to Air Missile System ChunGung (2012), developed by Agency for Defense Development, Internal White Paper.

NASA Systems Engineering Handbook and INCOSE Systems Engineering Handbook. 
NASA-STD-7009 (Standard for Models and Simulations) 2008.

National Computerization Agency, (1990), Verification, Validation and Test Guideline for Software.

Oberkampf, W. L., Trucano, T. G., and Hirsch, C. (2004), Verification, Validation, and Predictive Capability in Computational Engineering and Physics, Applied
Mechanics Reviews, 57(5), 345-384.

Tolk, A. (2012), Engineering Principles of Combat Modeling and Distributed Simulation, Wiley.

Wakeland, W., Shervais, S., and Raffo, D. (2005), Heuristic Optimization as a V\&V Tool for Software Process Simulation Models, Software Process: Improvement and Practice, 10(3), 301-309. 